\title{
Fibrosis as a Cause or a Consequence of White Adipose Tissue Inflammation in Obesity
}

\author{
Sophie Reggio • Vanessa Pellegrinelli • Karine Clément • Joan Tordjman
}

Published online: 30 December 2012

(C) Springer Science+Business Media New York 2012

\begin{abstract}
Obesity is defined as an excess accumulation of white adipose tissue associated with a low grade inflammation which is one contributor to obesity complications such as diabetes. Recently, we and others have demonstrated fibrotic depots in white adipose tissue of obese subjects. Fibrosis is characterized by an excessive accumulation of extracellular matrix components which could result in tissue dysfunction. Extracellular matrix is a fibrillar network composed of structural (collagens) and adhesion (fibronectin) proteins crucial for tissue architecture and biological pathway Histol Histopathol 27:15151528,2012 . In this review, we aim to describe two hypotheses trying to explain the sequence of events linking fibrosis, inflammation and obesity complications. In the first one, local adipose tissue hypoxia could induce fibrosis accumulation which in turn through adipocyte dysfunctions might provoke adipose tissue inflammation. In the
\end{abstract}

S. Reggio $\cdot$ V. Pellegrinelli $\cdot$ K. Clément $(\bowtie) \cdot J$. Tordjman $(\bowtie)$ INSERM U872, team 7, Nutriomique,

15 , rue de l'école de médecine,

75006 Paris, France

e-mail: karine.clement@psl.aphp.fr

e-mail: joan.tordjman@crc.jussieu.fr

S. Reggio

e-mail: sophie.reggio@crc.jussieu.fr

V. Pellegrinelli

e-mail: vanessa.pellegrinelli@crc.jussieu.fr

S. Reggio $\cdot$ V. Pellegrinelli $\cdot \mathrm{K}$. Clément $\cdot \mathrm{J}$. Tordjman

Centre de Recherche des Cordeliers, UMR S 872,

Université Pierre et Marie Curie - Paris 6, Paris 75006, France

S. Reggio $\cdot$ V. Pellegrinelli $\cdot$ K. Clément $\cdot J$. Tordjman

Université Paris Descartes, UMR S 872, Paris 75006, France

K. Clément $\cdot$ J. Tordjman

Institut Cardiométabolisme et Nutrition (ICAN) Pitié Salpetrière

Hospital, Paris 75013, France second one, fibrosis could be a result of local adipose tissue inflammation induced by tissue expansion.

Keywords Fibrosis $\cdot$ Inflammation $\cdot$ Hypoxia $\cdot$ Obesity complications $\cdot$ Kinetics $\cdot$ White adipose tissue

\section{Introduction}

Obesity is defined as an expansion of white adipose (WAT) tissue that is associated with low grade inflammation which possibly contributes to the development of health complications such as diabetes, liver pathologies, dyslipidemia, cardiovascular diseases as well as some cancers [2-4].

Classically the fat mass expansion results with an increase in the volume (hypertrophy) and the number (hyperplasia) of adipocytes $[5,6]$. These morphological changes associate with a myriad of effects including hypoxia, immune cell accumulation [7] and extracellular matrix (ECM) remodeling [8••]. In the past, numerous studies investigating WAT inflammation have shown that the release of pro-inflammatory cytokines, such as TNF $\alpha$, IL-6 or gp130 cytokines, are implicated in insulin-resistance $[9,10]$. However many more pro and antiinflammatory mediators produced by the adipose tissue have been described since then. A differential response in the development of obesity complications exists depending on WAT depot distribution. A proinflammatory pattern typically characterizes visceral WAT. Indeed the visceral WAT which is more inflamed with higher accumulation of macrophages [7] has more detrimental effects than the subcutaneous WAT [11]. However, inflammation is not the only factor contributing to WAT dysfunctions; the effects of hypoxia and fibrosis may also play a role in obesity complications via notably ectopiclipid accumulation in non-adipocyte cells. Indeed, WAT remodeling eventually associated with a limited capacity to adequately expand might induce lipid spill over and this 
triglyceride accumulation in other depots such as liver or muscle. However, lipid accumulation in non-specialized cells is deleterious and can contribute to cell death resulting in the development of pathology such as NASH (Non-Alcoholic Steatohepatitis) a liver injury associated with obesity [12].

In this review, we aim to describe the relationships and discussed sequence of events associated with hypoxia, ECM remodeling and inflammation in the development of obesity and associated complications. Whether fibrosis is a cause or consequence of inflammation is presently unknown but will be discussed further.

\section{Extracellular Matrix Remodeling and Fibrosis}

The extracellular matrix is essential for the architecture of the tissue and a myriad of associated biological functions. This fibrillar network is composed of many structural proteins, such as collagens, and adhesion proteins, including fibronectin and proteoglycans (perlecan, decorin) [13, 14]. Collagens are crucial for the ECM architecture and particularly collagen I, III and VI are key components in animal tissues. ECM components (i.e., fibronectin or collagens) directly interact with membrane receptors (i.e., integrins), particularly, by influencing cell behavior. Integrins are directly linked to the cytoskeleton. Thus any modification of ECM components can be transmitted via integrins and impact on cell movement during development and proliferation, differentiation, apoptosis.

ECM also modulates the bioavailability of many growth factors. For example proteoglycans sequester biomolecules such as growth factors (TGF- $\beta$, VEGF, FGF etc.) and MMPs (Matrix Metalloproteinases). MMPs are proteolytic enzymes highly specialized in the degradation of ECM component $[15,16]$ and each member of the MMP family is specifically involved in degradation of one or several ECM components. These enzymes are crucial to maintain ECM structure but also for the release of active molecules with various biological functions [16]. For example, the cleavage of collagen XVIII by MMP-9 releases the endostatin fragment [17] which has the ability to inhibit the formation of new blood vessels [18-20]. Scherrer et al. have also revealed that endotrophin, a collagen VI fragment, is implicated in fibrosis, inflammation and angiogenesis [21]. MMPs can also activate growth factors sequestered in the ECM. For example, inactive pro-TGF- $\beta$ interacts with fibronectin [22] and thrombospondin [23]. In the context of ECM remodeling, MMP-9 cleaves pro-TGF- $\beta$ which is rapidly released and activated [24]. The activated form of TGF- $\beta$ has been shown to be implicated in fibrogenesis [25] by promoting the synthesis of collagen I.

In pathological processes, several organs can be the site of fibrosis accumulation such as the liver, heart, and kidney
[26]. Fibrosis is indeed defined as an excessive accumulation of ECM components which is a result of degradation impairment and an excess synthesis of fibrillar components including collagen I, III and VI. The repair process is fundamental for the replacement of dead or injured cells in response to inflammation. This process starts during the regenerative phase where cells are replaced by others. However, if the damage persists, then myofibroblasts are activated and secrete collagens and fibrillar components, in order to replace the normal parenchymal tissue. As a result, fibrotic depots accumulate in tissues.

Thus, while tissue remodeling and fibrosis appearance is initially a physiological and beneficial process for the tissue; fibrosis accumulation sometimes associates with the absence of resolution of inflammation which can be can highly deleterious to organ biological function and homeostasis with time.

\section{WAT and Extracellular Matrix}

As all mammalian tissue, WAT is the site of a dynamic remodeling of the elements of the extracellular matrix. This fibrillar network composed of structural protein as collagen I, III and VI and adhesion protein (fibronectin) might play a crucial role in the physiology but also in the pathophysiology of the WAT.

\section{In Physiological Conditions}

WAT has a unique plasticity illustrated by its capacity to rapidly expand or to diminish in size to ensure proper systemic energy homeostasis. The first data on the WAT ECM was reported by Napolitano, in 1963, using Transmission Electron Microscopy. Napolitano examined the WAT development in rats and highlighted the presence of fibrillar collagen networks surrounding adipocytes [27]. The ECM can be remodeled in both physiological and pathological conditions. For example, a notable ECM remodeling for WAT function occurs during adipocyte differentiation. Indeed during the differentiation of adipose cell progenitors into mature adipocytes, the collagen I/fibronectin-rich matrix surrounding pre-adipocytes is progressively replaced by a basement membrane mainly composed of collagen IV, laminin, nidogen and perlecan. This remodeling is crucial for the conversion of fibroblast-like cell into an adipocyte, a spherical cell which store triglycerides in the lipid droplets influencing cell size. Thus, culturing 3T3-L1 preadipocytes on a fibronectin-rich matrix strongly inhibits adipocyte differentiation [28]. Conversely, human preadipocytes cultured on a matrigel (mainly composed of basement membrane components) or laminin (the main basement membrane component) display enhanced adipose differentiation [29]. 
At the tissue level, a human study performed in healthy and growing children, has shown the importance of collagen accumulation and described a strong negative correlation between the abundance of collagen deposition and adipocyte size [30]. Taken together, these studies emphasize the crucial role of ECM in adipose tissue physiology and development.

\section{In Pathological Conditions}

Impairment in ECM organization and composition can lead to important WAT dysfunctions. A chronic positive energy balance promoting obesity development induces WAT modifications with adipocyte hypertrophy and hyperplasia, accumulation of inflammatory cells and neovascularization [31]. These morphological modifications lead to ECM remodeling with degradation of the existing ECM and the production of new ECM components. WAT remodeling has been shown in mice consuming a high fat diet for 20 weeks [32]. Strissel et al. have observed an acute collagen deposition associated with an increase of inflammatory markers and the concomitant accumulation of macrophages in WAT.
The examination of the transcriptomic signature of obese WAT has highlighted for the first time the over-expression of many ECM components in obese compared to lean WAT $[33,34]$. ECM gene networks were tidily limited with inflammatory gene networks in this set of experiments. Based on the evidence of major expression changes in ECM components, our team has measured the abundance of collagen depots in obese subjects compared to lean ones $[8 \cdot \bullet]$. The red picrosirius staining quantification, specific for fibrillar collagens, showed higher collagen deposition in both obese scWAT and visceral WAT compared to lean adipose tissues (Fig. 1). Periadipocyte accumulation of collagens was particularly characteristic of obesity. Our team has also characterized the collagen deposition and observed an increase in the deposition of collagen I, III and VI in obese individuals.

While accumulation of collagen appears as an important feature in obese WAT, the precise origin of fibrosis accumulation is scarcely understood. Preadipocytes are considered as candidate cells that may play a crucial role in WAT remodeling. Indeed cultures of human preadipocytes with pro-inflammatory macrophages media induces an over-expression and secretion
Fig. 1 Adipose tissue fibrosis in obesity. Red picrosirius staining of a subcutaneous white adipose tissue (WAT) and b visceral WAT of lean subjects compared to c, e subcutaneous WAT and $\mathbf{d}$ visceral WAT of obese subjects. Black arrows $=$ collagen depots
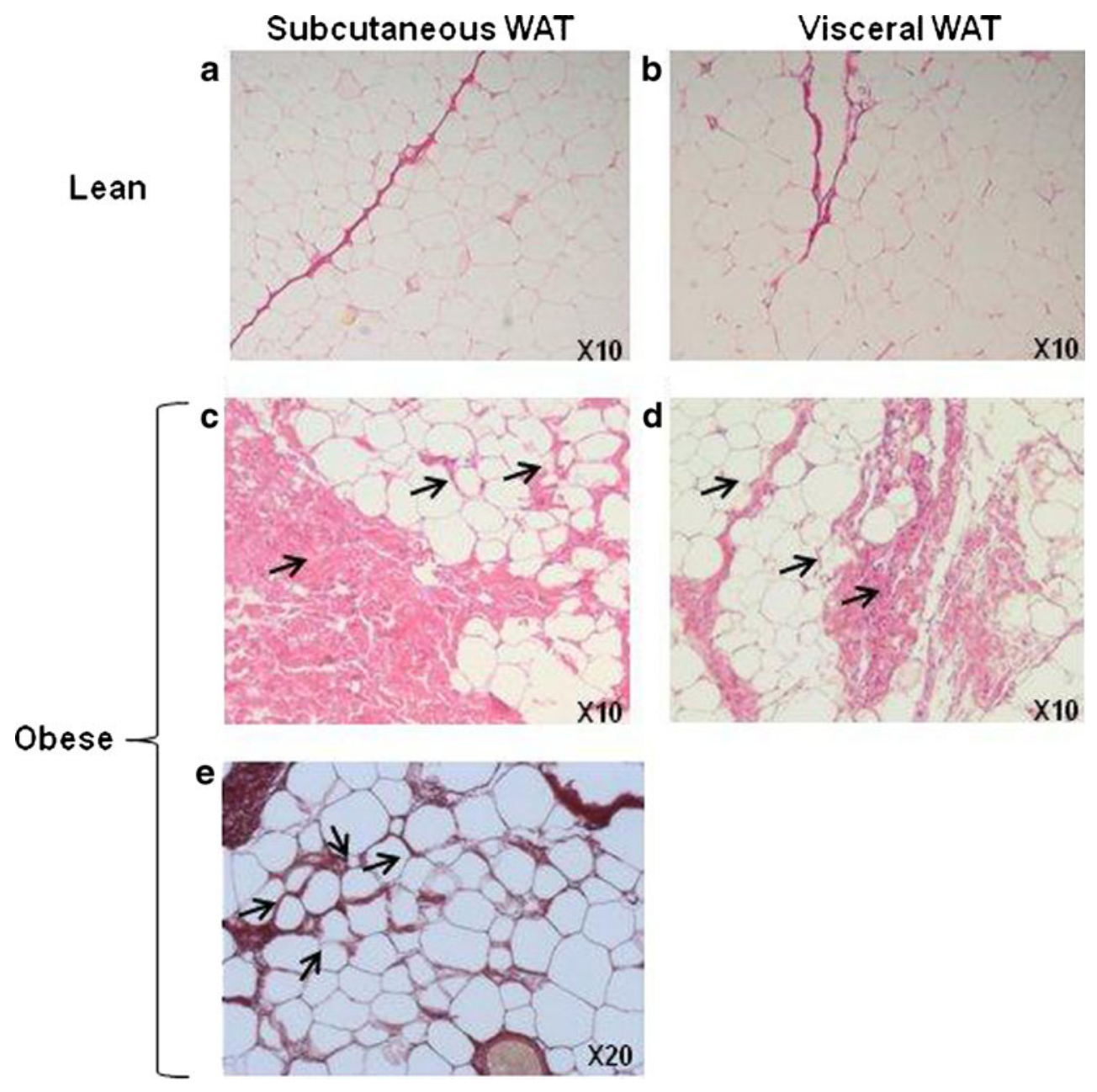

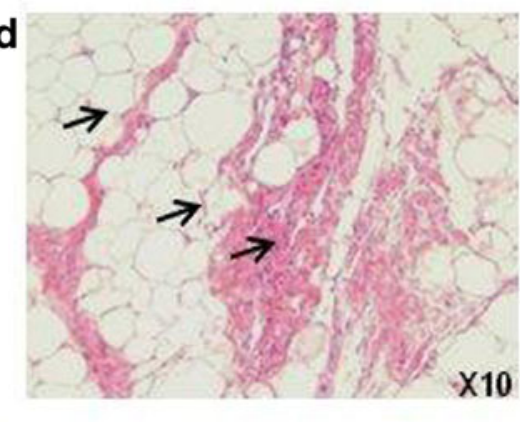


of several collagen types [35]. However, other cell types may also play a role in the accumulation of WAT fibrosis. For example, endothelial cells as epithelial cells can be converted into myofibroblasts via a process named endothelialmesenchymal transition in many tissues [36]. This specific process has not been described in the adipose tissue. Pericytes, the mural cells of blood vessels, provide structural and nutritional support to endothelial cells and also participate to tissue fibrogenesis. In a pathological context, pericytes can detach from the vessel walls, migrate and acquire a fibroblast-like morphology to secrete collagens. This phenomenon has been described in several fibrotic diseases such as in liver [37] and kidney injuries [38] as well as systemic sclerosis [39]. In human WAT, pericytes are poorly characterized but new evidence demonstrated their activation with increased expression of collagen I and TGF $\beta$ (V. Pellegrinelli, personal data). Furthermore, endothelial cells in obese visceral WAT subjects were reported to be senescent [40] inducing vascular wall remodeling as a pericyte detachment which could be converted in fibroblast-like cell.

Fibrocytes, a population of fibroblast-like cells present in blood, could also contribute to the growth and maintenance of adipose tissue [41] and their lineage commitment is regulated by TGF- $\beta$. Furthermore, it has been shown that fibrocytes participate in wound healing after injury [42]. Thus, during obesity, fibrocytes could invade adipose tissue and actively participate in WAT fibrosis.

Finally, adipocytes could also contribute to the production of fibrosis based on our recent observations showing the presence of peri-cellular fibrosis surrounding adipocytes [8••], which can be due to an increased synthesis or low degradation of ECM components by the adipocytes. However, cellular actors implicated in WAT fibrosis are poorly defined and more studies are warranted for their characterization. Fibrosis seems implicated in WAT dysfunction and obesity complications. Bioinformatics analysis of WAT transcriptome has demonstrated a strong positive correlation between BMI, WAT inflammation and ECM components [33]. Furthermore, ob/ob mice lacking collagen VI in WAT have significant improvements in their glucose metabolism which is associated with the absence of fibrotic depots and inflammation [43•]. This result highlights the implication of WAT fibrosis in insulin-resistance which has been supported by another study performed in human subjects in which collagen VI expression strongly correlated with glucose metabolism impairment [44•]. Overall, these studies suggest that obesity, WAT inflammation and fibrosis can sometimes be closely related events but the sequences of the events remains to be determined. On the other hand, data reported that overfeeding in healthy men for 2 months leads to collagen deposition and neovascularization in scWAT, with no changes in immune cell accumulation and inflammation suggesting that inflammation and collagen accumulation might not be always linked [45].

\section{The First Hypothesis: Fibrosis as a Cause of WAT Inflammation}

The first hypothesis proposes fibrosis as an early event in obesity and related metabolic disorders such as insulin resistance and a cause of WAT inflammation. This hypothesis is related to experimental data mostly in animal models linking the appearance of adipose tissue hypoxia and the promotion of fibrosis. In such conditions, hypoxia and fibrosis could lead to an adipocyte dysfunction which in turn might induce inflammation as detailed below (Fig. 2).

Indeed in order to provide oxygen and nutrients to adipocytes, the WAT vascularization is highly developed and each adipocyte is in close contact with capillaries [46]. A very functional vascular network is thus critical for adipose tissue homeostasis. The expansion of WAT in obesity should be accompanied by a proportional increase in the vascularization, in order to provide adequate supply in oxygen and nutrients to the tissue. However, several studies have suggested abnormal oxygen supply in obese WAT for example by measuring the Adipose Tissue Blood Flow (ATBF) or the AT oxygen partial pressure. Using radioactive xenon, a first report in 1966 has demonstrated a reduction in the radioisotope clearance in obese scWAT [47]. Several studies performed in animal models and in humans have confirmed this result. For example, ATBF is reduced in Sprague-Dawley rats using radio-labeled microsphere [48]. More recently, a study using polarographic electrode to measure the subcutaneous tension oxygen has shown that obese scWAT was hypoxic [49]. However, while hypoxia could be associated with obesity development, it does not necessarily associate with insulin-resistance. In a recent study, ATBF was reduced only in obese diabetic Zucker rats and not in non-obese diabetic Goto-Kakizaki rats. However, in another study in humans, the ATBF reduction correlates with the insulinsensitivity state of the subjects $[50,51]$.

Molecular regulators of hypoxia are better understood. The HIF1 proteins regulate the response to oxygen availability at the transcriptional level [52]. HIF1 is also tightly regulated at the protein levels. During hypoxia, HIF1 protein is stabilized and can regulate its main target genes which are pro-angiogenic genes such as VEGF A and angiopoietin-2. It has been proposed in several conditions such as metabolic syndrome and diabetes that HIF pathway could be dysregulated [53]. As such the gene expression of HIF1a is up-regulated in scWAT of obese subjects [54]. Immunodetection of HIF $1 \alpha$ in ob/ob mice adipose tissue has showed that adipocytes are the cells predominantly expressing HIF1a in WAT [55]. Thus disturbances in HIF1 signaling seem to play a detrimental role in adipocyte function $[56 \cdot, 57,58]$. Importantly, KO mice targeted for HIF1a adipocytes are protected from the consequence of HFD [57, 58]. Adipocytes secrete angiogenic factors, such as leptin, 
Fig. 2 Fibrosis as a cause or consequence of WAT inflammation. This first hypothesis proposes that during weight gain, hypoxia enhances adipose tissue fibrosis that could lead to an adipocyte dysfunction which in turn might induce inflammation. The second hypothesis proposes fibrosis as a late event in obesity development and as a consequence of low-grade inflammation

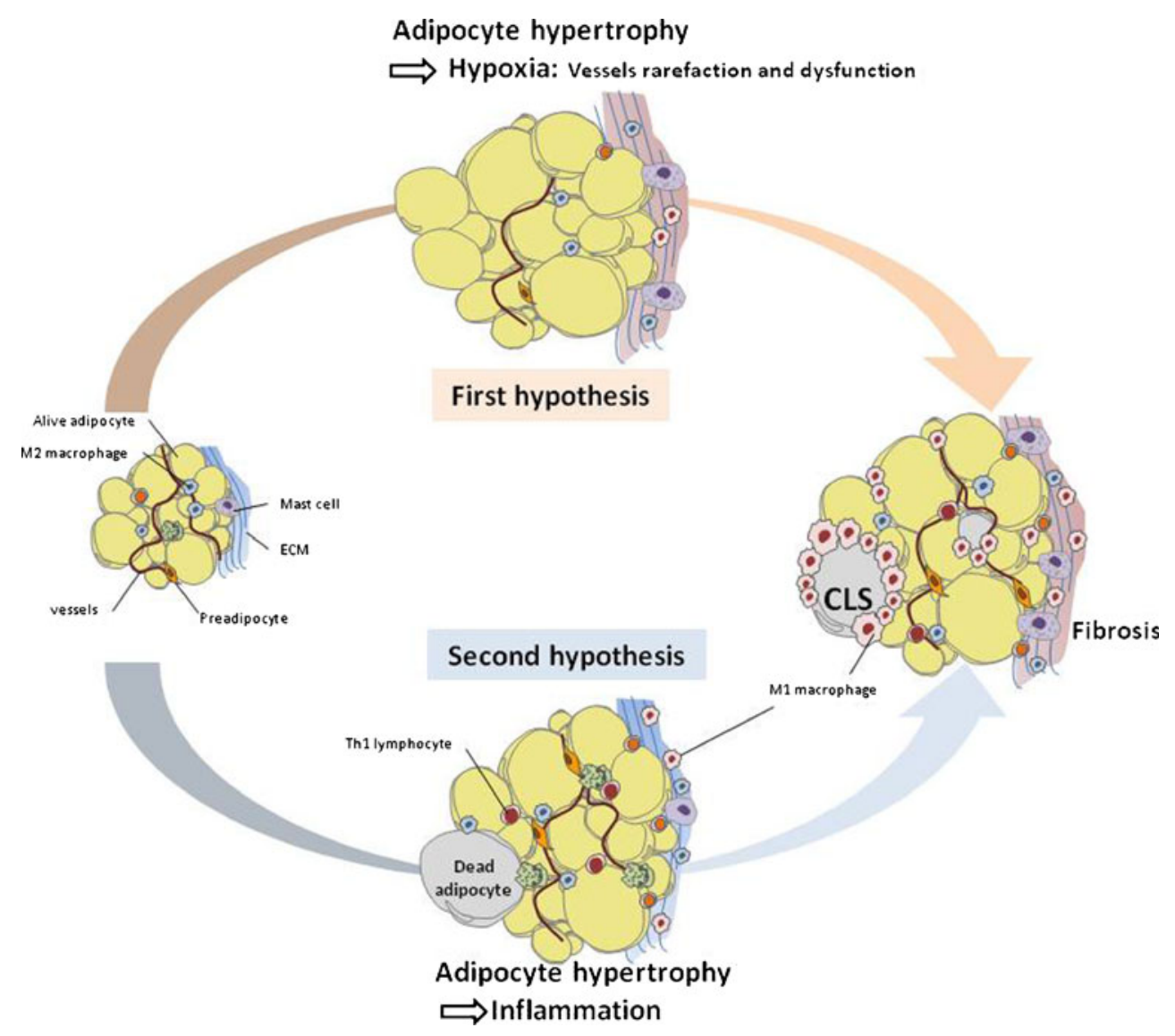

adiponectin, angiopoietin-2 and VEGF A [46], which expression is stimulated by HIF1. HFD fed mice overexpressing VEGF A in WAT have also an increase of vessel density and display an improvement of their glucose and lipid metabolism [59]. Overall, these observations suggest a determinant role of oxygen supply as well as the involvement of HIF-related molecular mechanisms in WAT homeostatic function. Furthermore it has been shown that local hypoxia and HIF dysregulation could contribute to fibrosis. In addition to pro-angiogenic genes, the over-expression of HIF1 in mouse WAT induces a significant up regulation of many pro-fibrotic genes such as collagen I, III, VI, CTGF (connective tissue growth factor) and LOX (Lysyl Oxydase) associated with fibrosis appearance. LOX is an enzyme crucial for collagen cross-linking and its overexpression has been shown to be associated in several organs fibrosis [60-62] and regulated by hypoxia [63]. These mice overexpressing HIF $1 \alpha$ showed impairment in glucose metabolism [56•]. LOX seemed to be a crucial component for the formation of WAT fibrosis because the pharmacological inhibition of LOX activity in mice overexpressing HIF1 was associated with a reduction in size of the collagen-laden streaks in scWAT. In such condition glucose metabolism was improved.

The increase in LOX activity induces a collagen crosslinking and thus tissue stiffening. This rigid fibrotic deposition surrounding adipocytes could create hypoxic pockets and increase mechanical stress by expanding adipocytes and leading to necrosis. In such condition, adipocyte deaths create a local inflammation that results in the accumulation of inflammatory cells dedicated initially to remove cellular debris. This phenomenon occurs in chronic inflammation and could self-perpetuate and contribute to the development of insulin resistance.

However, others studies have shown that HIF $1 \alpha$ can also activate some inflammatory genes such as the transcription factor NFKB [64] and thus hypoxia could directly induce WAT inflammation without fibrosis. During obesity, the importance of adipocyte necrosis has not been elucidated and the measure of adipocyte turnover. Indeed, analyses of the integration of $14 \mathrm{C}$ derived from nuclear bomb tests in genomic DNA of WAT [65], have not shown any modification in the adipocyte death or generation rate in obese WAT. As a result, the time-course of events seem to be more complicated than expected, especially in the natural progression of obesity in humans.

\section{The Second Hypothesis: Fibrosis as a Consequence of WAT Inflammation}

This hypothesis proposes fibrosis as a late event in obesity development and as a consequence of low-grade inflammation 
(Fig. 2). WAT is a crucial endocrine organ which can secrete many adipocytokines such as leptin, [CC] Chemokines Ligand 2 (CCL-2), IL-6 (Interleukine-6) and adiponectin which are implicated in physiological and pathological processes [66-68]. Obesity is classically associated with chronic and low-grade inflammatory status characterized by immune cell accumulation such as macrophages [7], mast cells [69], neutrophils [70] and T lymphocytes [71] which may relate to the development of obesity-induced insulin-resistance $[2,66]$. It has been shown that macrophage accumulation precedes or is associated with the development of insulin-resistance in obese animals $[72,73]$. The invalidation of genes involved in macrophage recruitment (CCR2 CC Chemokine receptor 2, the CCL2 receptor), inflammatory cytokine production (TNF $\alpha)$, and pro-inflammatory transcriptional activation $(\mathrm{NF} K \mathrm{~B})$ in several mouse models have revealed a protective effect to a high-fat diet inducing insulin-resistance [72, 74]. Macrophages can exist in different activation states with distinct properties [75] (i.e., pro-inflammatory M1 macrophages and antiinflammatory M2 macrophages). M1 macrophages are crucial for the initial steps of the immune response in order to eliminate pathogens. Conversely, M2 macrophages appear to be important for the final step of the immune response to replace the injured tissue by connective tissue through secretion of profibrotic molecules such as TGF- $\beta$ or MMP9. Numerous studies have tried to phenotype adipose tissue macrophages (ATMs). However the characterization of the precise phenotype of ATMs remains challenging due to the lack of specific markers for each class of macrophages. Histological analysis have revealed the presence of M1 macrophages in structure named "crown-like structure" surrounding necrotic adipocytes in human and mice [76]. Another study has highlighted the presence of M2 macrophages in the interstitial space between adipocytes in WAT mice [77]. In human WAT, the characterization is more complex and a mixed M1/M2 phenotype has been demonstrated [78] and notably in fibrotic depots [8••]. The presence in WAT of macrophages, mast cells and T lymphocytes participate to reinforce the WAT inflammatory state by secreting cytokines such as TNF $\alpha$, IFN $\gamma$ and IL-6; Tlymphocytes are also involved in the development of fibrosis by releasing TGF- $\beta$ and MMP9. Interestingly, mast cells particularly accumulate in fibrotic WAT depots [69] in humans, but their precise role in the promotion or degradation of fibrosis in human WAT is unknown.

Activin A, is a TGF- $\beta$ family protein that has profibrotic proprieties [25]. Smad 2 and 3 mediate the pathway of the TGF- $\beta$ family by binding to the collagen I promoter [79]. Furthermore, neutrophils [80] and macrophages [81] are known to secrete MMP9 which have been shown crucial for the activation of the TGF- $\beta$ family member [24]. In obese subjects, MMP9 and Activin A have been shown to be over-expressed in WAT $[82,83]$ and have increased systemic levels [84] (M. Keophiphath, unpublished data). Furthermore, our team has recently shown an activin A over-expression in inflammatory preadipocytes [85] which then can act on cells via the Smads pathway by inducing collagen expressions.

In this hypothesis, inflammation is due to the accumulation of immune cells as an early event in obesity development. The secretion by immune cells of pro-fibrotic factors such as activin A and MMP9 are both crucial for ECM remodeling leading to fibrosis.

Recently, using a time-course microarray study during a diet-induced obesity in mice, Kwon et al. have revealed that inflammatory related-genes are over-expressed in visceral WAT prior to genes being selected for fibrosis formation [86]. This result seems to validate the hypothesis that fibrosis is a late event in obesity development and influenced by local adipose tissue inflammation.

\section{Conclusion}

There is a close relationship between WAT inflammation and fibrosis [2] with accumulating evidence indicating that WAT dysfunction contributes to obesity associated complications [87, 88]. However, the time-course of the events is not yet fully established. Different studies have generated two main hypotheses. The first hypothesis proposes that local adipose tissue hypoxia induces fibrosis promoting adipocyte necrosis in order to renew the cells. Subsequently, in order to remove cellular debris, immune cells, such as macrophages and $\mathrm{T}$ lymphocytes, are recruited and induce a low-grade inflammation which can self-perpetuate over time. The second hypothesis proposes that adipose tissue expansion produces immune cell accumulation which elicits a low-grade inflammation. Fibrosis is defined as a process induced in the context of an unresolved chronic inflammation [26]. Therefore, the accumulation of immune cells, by secreting pro-fibrotic molecules, which induces the activation of fibrosis-producing cells and also the removal of dead cells, could participate in fibrosis accumulation.

One of the hypotheses concerning the link between obesity and type 2 diabetes proposes that insulin-resistance is induced by pro-inflammatory cytokines such as TNF $\alpha$ and IL-6 [89]. However, Vidal-Puig and Virtue have proposed a new theory, "the adipose tissue expendability ", based on the concept that subcutaneous WAT has a limited capacity to expand [90]. When WAT storage is exceeded mechanical limitations such as ECM remodeling could occur. Thus, in this hypothesis, WAT fibrosis limits adipose tissue expansion, thereby; excess lipids cannot store anymore and are directed to ectopic sites such as liver and muscle. Then in these tissues, lipids exert toxic effects and induce insulin-resistance. Currently, this theory has not yet been fully confirmed but supportive evidence is in line with the "adipose tissue expendability theory". 
Mice deleted for collagen VI, one of the main collagen implicated in WAT fibrosis, on an ob/ob background have more fat mass but develop less insulin-resistance [43•]. Furthermore, a human study has revealed a positive correlation between collagen VI expression in scWAT and glucose metabolism impairment [44•].

Finally, the sequence of events such as WAT hypoxia, inflammation and fibrosis leading to obesity complications are poorly understood and more kinetic studies on obesity development, notably in humans such as that performed in [86], are necessary to understand the development of obesity complications.

Acknowledgment We are grateful to colleagues at U872 team 7 particularly for helpful discussions. We thank Assistance Publique/Hôpitaux de Paris (APHP), National Research Agency (ANR Adipofib, PHRC fibrota, IHU), Fondation pour la Recherche Medicale (FRM), and the corporate Echosens, which support our studies on WAT fibrosis. J. Tordjman and K. Clément equally contributed to this manuscript.

Disclosure No potential conflicts of interest relevant to this article were reported.

\section{References}

Papers of particular interest, published recently, have been highlighted as:

- Of importance

•- Of major importance

1. Catalán V, Gómez-Ambrosi J, Rodríguez A, Frühbeck G. Role of extracellular matrix remodelling in adipose tissue pathophysiology. Relevance in the development of obesity. Histol Histopathol. 2012;27:1515-28.

2. Hotamisligil GS. Inflammation and metabolic disorders. Nature. 2006;444:860-7.

3. Trayhurn P, Wood IS. Adipokines: inflammation and the pleiotropic role of white adipose tissue. Br J Nutr. 2004;92:347-55.

4. Lumeng $C N$, Saltiel AR. Inflammatory links between obesity and metabolic disease. J Clin Invest. 2011;121:2111-7.

5. Rosen ED, MacDougald OA. Adipocyte differentiation from the inside out. Nat Rev Mol Cell Biol. 2006;7:885-96.

6. Sethi JK, Vidal-Puig AJ. Thematic review series: adipocyte biology. Adipose tissue function and plasticity orchestrate nutritional adaptation. J Lipid Res. 2007;48:1253-62.

7. Cancello R, Tordjman J, Poitou C, Guilhem G, Bouillot JL, Hugol $\mathrm{D}$, et al. Increased infiltration of macrophages in omental adipose tissue is associated with marked hepatic lesions in morbid human obesity. Diabetes. 2006;55:1554-61.

8. •- Divoux A, Tordjman J, Lacasa D, Veyrie N, Hugol D, Aissat A, et al. Fibrosis in human adipose tissue: composition, distribution, and link with lipid metabolism and fat mass loss. Diabetes. 2010;59:2817-25. This study highlights fibrosis in the WAT of obese subject and notably some fibrillar collagen such as the type I, III and VI.

9. Permana PA, Menge C, Reaven PD. Macrophage-secreted factors induce adipocyte inflammation and insulin resistance. Biochem Biophys Res Commun. 2006;341:507-14.
10. Marcos-Gómez B, Bustos M, Prieto J, Martínez JA, MorenoAliaga MJ. Obesity, inflammation and insulin resistance: role of gp 130 receptor ligands. An Sist Sanit Navar. 2008;31:113-23.

11. Kissebah AH, Krakower GR. Regional adiposity and morbidity. Physiol Rev. 1994;74:761-811.

12. Ibrahim SH, Kohli R, Gores GJ. Mechanisms of lipotoxicity in NAFLD and clinical implications. J Pediatr Gastroenterol Nutr. 2011;53:131-40.

13. Hynes RO. The extracellular matrix: not just pretty fibrils. Science. 2009;326:1216-9.

14. Hynes RO, Naba A. Overview of the matrisome-an inventory of extracellular matrix constituents and functions. Cold Spring Harb Perspect Biol. 2012;4:a004903.

15. Martins VL, Caley M, O’Toole EA. Matrix metalloproteinases and epidermal wound repair. Cell Tissue Res. 2012. doi:10.1007/ s00441-012-1410-z.

16. Sternlicht MD, Werb Z. How matrix metalloproteinases regulate cell behavior. Annu Rev Cell Dev Biol. 2001;17:463-516.

17. Nilsson UW, Dabrosin C. Estradiol and tamoxifen regulate endostatin generation via matrix metalloproteinase activity in breast cancer in vivo. Cancer Res. 2006;66:4789-94.

18. Marneros AG, Keene DR, Hansen U, Fukai N, Moulton K, Goletz $\mathrm{PL}$, et al. Collagen XVIII/endostatin is essential for vision and retinal pigment epithelial function. EMBO J. 2004;23:89-99.

19. Marneros AG, Olsen BR. Physiological role of collagen XVIII and endostatin. FASEB J. 2005;19:716-28.

20. Zatterstrom UK, Felbor U, Fukai N, Olsen BR. Collagen XVIII/ endostatin structure and functional role in angiogenesis. Cell Struct Funct. 2000;25:97-101.

21. Park J, Scherer PE. Adipocyte-derived endotrophin promotes malignant tumor progression. J Clin Invest. 2012. doi:10.1172/ JCI63930.

22. Mooradian DL, Lucas RC, Weatherbee JA, Furcht LT. Transforming growth factor-beta 1 binds to immobilized fibronectin. J Cell Biochem. 1989;41:189-200.

23. Schultz-Cherry S, Murphy-Ullrich JE. Thrombospondin causes activation of latent transforming growth factor-beta secreted by endothelial cells by a novel mechanism. J Cell Biol. 1993;122:92332.

24. Yan J-D, Yang S, Zhang J, Zhu T-H. BMP6 reverses TGF-beta1induced changes in HK-2 cells: implications for the treatment of renal fibrosis. Acta Pharmacol Sin. 2009;30:994-1000.

25. Biernacka A, Dobaczewski M, Frangogiannis NG. TGF- $\beta$ signaling in fibrosis. Growth Factors. 2011;29:196-202.

26. Wynn TA. Common and unique mechanisms regulate fibrosis in various fibroproliferative diseases. J Clin Invest. 2007;117:5249.

27. Napolitano L. The differentiation of white adipose cells. An electron microscope study. J Cell Biol. 1963;18:663-79.

28. Spiegelman BM, Ginty CA. Fibronectin modulation of cell shape and lipogenic gene expression in 3T3-adipocytes. Cell. 1983;35:65766.

29. O'Connor KC, Song H, Rosenzweig N, Jansen DA. Extracellular matrix substrata alter adipocyte yield and lipogenesis in primary cultures of stromal-vascular cells from human adipose. Biotechnol Lett. 2003;25:1967-72.

30. Tam CS, Tordjman J, Divoux A, Baur LA, Clément K. Adipose tissue remodeling in children: the link between collagen deposition and age-related adipocyte growth. J Clin Endocrinol Metab. 2012;97:1320-7.

31. Rutkowski JM, Davis KE, Scherer PE. Mechanisms of obesity and related pathologies: the macro- and microcirculation of adipose tissue. FEBS J. 2009;276:5738-46.

32. Strissel KJ, Stancheva Z, Miyoshi H, Perfield 2nd JW, DeFuria J, Jick Z, et al. Adipocyte death, adipose tissue remodeling, and obesity complications. Diabetes. 2007;56:2910-8. 
33. Mutch DM, Tordjman J, Pelloux V, Hanczar B, Henegar C, Poitou $\mathrm{C}$, et al. Needle and surgical biopsy techniques differentially affect adipose tissue gene expression profiles. Am J Clin Nutr. 2009;89:517.

34. Henegar C, Tordjman J, Achard V, Lacasa D, Cremer I, Guerre-Millo $\mathrm{M}$, et al. Adipose tissue transcriptomic signature highlights the pathological relevance of extracellular matrix in human obesity. Genome Biol. 2008;9:R14.

35. Lacasa D, Taleb S, Keophiphath M, Miranville A, Clement K. Macrophage-secreted factors impair human adipogenesis: involvement of proinflammatory state in preadipocytes. Endocrinology. 2007;148:868-77.

36. Potenta S, Zeisberg E, Kalluri R. The role of endothelial-tomesenchymal transition in cancer progression. $\mathrm{Br} \mathrm{J}$ Cancer. 2008;99:1375-9.

37. Reynaert H, Thompson MG, Thomas T, Geerts A. Hepatic stellate cells: role in microcirculation and pathophysiology of portal hypertension. Gut. 2002;50:571-81.

38. Lin S-L, Kisseleva T, Brenner DA, Duffield JS. Pericytes and perivascular fibroblasts are the primary source of collagenproducing cells in obstructive fibrosis of the kidney. Am J Pathol. 2008; 173:1617-27.

39. Rajkumar VS, Sundberg C, Abraham DJ, Rubin K, Black CM. Activation of microvascular pericytes in autoimmune Raynaud's phenomenon and systemic sclerosis. Arthritis Rheum. 1999;42:930 41.

40. Villaret A, Galitzky J, Decaunes P, Estève D, Marques M-A, Sengenès $\mathrm{C}$, et al. Adipose tissue endothelial cells from obese human subjects: differences among depots in angiogenic, metabolic, and inflammatory gene expression and cellular senescence. Diabetes. 2010;59:2755-63.

41. Hong KM, Burdick MD, Phillips RJ, Heber D, Strieter RM. Characterization of human fibrocytes as circulating adipocyte progenitors and the formation of human adipose tissue in SCID mice. FASEB J. 2005;19:2029-31.

42. Bucala R, Spiegel LA, Chesney J, Hogan M, Cerami A. Circulating fibrocytes define a new leukocyte subpopulation that mediates tissue repair. Mol Med. 1994;1:71-81.

43. - Khan T, Muise ES, Iyengar P, Wang ZV, Chandalia M, Abate N, et al. Metabolic dysregulation and adipose tissue fibrosis: role of collagen VI. Mol Cell Biol. 2009;29:1575-91. This study demonstrates in a mouse model the crucial role of collagen VI in WAT fibrosis and metabolism disorders.

44. - Pasarica M, Gowronska-Kozak B, Burk D, Remedios I, Hymel $\mathrm{D}$, Gimble J, et al. Adipose tissue collagen VI in obesity. J Clin Endocrinol Metab. 2009;94:5155-62. This study shows the strong correlation between WAT fibrosis characterized by collagen VI expression and insulin-resistance in humans.

45. Alligier M, Meugnier E, Debard C, Lambert-Porcheron S, Chanseaume E, Sothier M, et al. Subcutaneous adipose tissue remodeling during the initial phase of weight gain induced by overfeeding in humans. J Clin Endocrinol Metab. 2012;97:E183-92.

46. Cao Y. Adipose tissue angiogenesis as a therapeutic target for obesity and metabolic diseases. Nat Rev Drug Discov. 2010;9:10715

47. Larsen OA, Lassen NA, Quaade F. Blood flow through human adipose tissue determined with radioactive xenon. Acta Physiol Scand. 1966;66:337-45.

48. Crandall DL, Goldstein BM, Huggins F, Cervoni P. Adipocyte blood flow: influence of age, anatomic location, and dietary manipulation. Am J Physiol. 1984;247:R46-51.

49. Kabon B, Nagele A, Reddy D, Eagon C, Fleshman JW, Sessler DI, et al. Obesity decreases perioperative tissue oxygenation. Anesthesiology. 2004;100:274-80.

50. Jansson PA, Larsson A, Lönnroth PN. Relationship between blood pressure, metabolic variables and blood flow in obese subjects with or without non-insulin-dependent diabetes mellitus. Eur J Clin Invest. 1998;28:813-8.

51. Karpe F, Fielding BA, Ilic V, Macdonald IA, Summers LKM, Frayn KN. Impaired postprandial adipose tissue blood flow response is related to aspects of insulin sensitivity. Diabetes. 2002;51:2467-73.

52. Rocha S. Gene regulation under low oxygen: holding your breath for transcription. Trends Biochem Sci. 2007;32:389-97.

53. Semenza GL. Hypoxia-inducible factor 1: oxygen homeostasis and disease pathophysiology. Trends Mol Med. 2001;7:345-50.

54. Cancello R, Henegar C, Viguerie N, Taleb S, Poitou C, Rouault C, et al. Reduction of macrophage infiltration and chemoattractant gene expression changes in white adipose tissue of morbidly obese subjects after surgery-induced weight loss. Diabetes. 2005;54:2277-86.

55. Higami Y, Barger JL, Page GP, Allison DB, Smith SR, Prolla TA, et al. Energy restriction lowers the expression of genes linked to inflammation, the cytoskeleton, the extracellular matrix, and angiogenesis in mouse adipose tissue. J Nutr. 2006;136:343-52.

56. - Halberg N, Khan T, Trujillo ME, Wernstedt-Asterholm I, Attie $\mathrm{AD}$, Sherwani $\mathrm{S}$, et al. Hypoxia-inducible factor 1alpha induces fibrosis and insulin resistance in white adipose tissue. Mol Cell Biol. 2009;29:4467-83. This study shows in mice models the implication of HIF1, the hypoxic transcription factor, WAT fibrogenesis in the context of obesity.

57. Jiang C, Qu A, Matsubara T, Chanturiya T, Jou W, Gavrilova O, et al. Disruption of hypoxia-inducible factor 1 in adipocytes improves insulin sensitivity and decreases adiposity in high-fat diet-fed mice. Diabetes. 2011;60:2484-95.

58. Krishnan J, Danzer C, Simka T, Ukropec J, Walter KM, Kumpf S, et al. Dietary obesity-associated Hifl $\alpha$ activation in adipocytes restricts fatty acid oxidation and energy expenditure via suppression of the Sirt2-NAD+ system. Genes Dev. 2012;26:259-70.

59. Sun K, Wernstedt Asterholm I, Kusminski CM, Bueno AC, Wang ZV, Pollard JW, et al. Dichotomous effects of VEGF-A on adipose tissue dysfunction. Proc Natl Acad Sci U S A. 2012;109:5874-9.

60. Decitre M, Gleyzal C, Raccurt M, Peyrol S, Aubert-Foucher E, Csiszar K, et al. Lysyl oxidase-like protein localizes to sites of de novo fibrinogenesis in fibrosis and in the early stromal reaction of ductal breast carcinomas. Lab Invest. 1998;78:143-51.

61. López B, González A, Hermida N, Valencia F, de Teresa E, Díez J. Role of lysyl oxidase in myocardial fibrosis: from basic science to clinical aspects. Am J Physiol Heart Circ Physiol. 2010;299:H1-9.

62. Szauter KM, Cao T, Boyd CD, Csiszar K. Lysyl oxidase in development, aging and pathologies of the skin. Pathol Biol. 2005;53:44856.

63. Erler JT, Giaccia AJ. Lysyl oxidase mediates hypoxic control of metastasis. Cancer Res. 2006;66:10238-41.

64. Barnes PJ, Karin M. Nuclear factor-kappaB: a pivotal transcription factor in chronic inflammatory diseases. N Engl J Med. 1997;336:1066-71.

65. Spalding KL, Arner E, Westermark PO, Bernard S, Buchholz BA, Bergmann $\mathrm{O}$, et al. Dynamics of fat cell turnover in humans. Nature. 2008;453:783-7.

66. Berg AH, Scherer PE. Adipose tissue, inflammation, and cardiovascular disease. Circ Res. 2005;96:939-49.

67. Kadowaki T, Yamauchi T, Kubota N, Hara K, Ueki K. Adiponectin and adiponectin receptors in obesity-linked insulin resistance. Novartis Found Symp. 2007;286:164-76. discussion 176-182;200-203.

68. Matsuzawa Y, Funahashi T, Nakamura T. Molecular mechanism of metabolic syndrome $\mathrm{X}$ : contribution of adipocytokines adipocytederived bioactive substances. Ann N Y Acad Sci. 1999;892:14654.

69. Divoux A, Moutel S, Poitou C, Lacasa D, Veyrie N, Aissat A, et al. Mast cells in human adipose tissue: link with morbid obesity, 
inflammatory status, and diabetes. J Clin Endocrinol Metab. 2012;97:E1677-85.

70. Shah TJ, Leik CE, Walsh SW. Neutrophil infiltration and systemic vascular inflammation in obese women. Reprod Sci. 2010;17:11624.

71. Wu H, Ghosh S, Perrard XD, Feng L, Garcia GE, Perrard JL, et al. $\mathrm{T}$-cell accumulation and regulated on activation, normal $\mathrm{T}$ cell expressed and secreted upregulation in adipose tissue in obesity. Circulation. 2007;115:1029-38.

72. Weisberg SP, Hunter D, Huber R, Lemieux J, Slaymaker S, Vaddi $\mathrm{K}$, et al. CCR2 modulates inflammatory and metabolic effects of high-fat feeding. J Clin Invest. 2006;116:115-24.

73. Xu H, Barnes GT, Yang Q, Tan G, Yang D, Chou CJ, et al. Chronic inflammation in fat plays a crucial role in the development of obesity-related insulin resistance. J Clin Invest. 2003;112:182130 .

74. Arkan MC, Hevener AL, Greten FR, Maeda S, Li Z-W, Long JM, et al. IKK-beta links inflammation to obesity-induced insulin resistance. Nat Med. 2005;11:191-8.

75. Martinez FO, Sica A, Mantovani A, Locati M. Macrophage activation and polarization. Front Biosci. 2008;13:453-61.

76. Cinti S, Mitchell G, Barbatelli G, Murano I, Ceresi E, Faloia E, et al. Adipocyte death defines macrophage localization and function in adipose tissue of obese mice and humans. J Lipid Res. 2005;46:2347-55.

77. Lumeng CN, DelProposto JB, Westcott DJ, Saltiel AR. Phenotypic switching of adipose tissue macrophages with obesity is generated by spatiotemporal differences in macrophage subtypes. Diabetes. 2008;57:3239-46.

78. Zeyda M, Farmer D, Todoric J, Aszmann O, Speiser M, Györi G, et al. Human adipose tissue macrophages are of an anti-inflammatory phenotype but capable of excessive pro-inflammatory mediator production. Int J Obes (Lond). 2007;31:1420-8.

79. Chen SJ, Yuan W, Mori Y, Levenson A, Trojanowska M, Varga J. Stimulation of type I collagen transcription in human skin fibroblasts by TGF-beta: involvement of Smad 3. J Invest Dermatol. 1999;112:49-57.
80. Tracy Jr TF. Editorial: Acute pancreatitis and neutrophil gelatinase MMP9: don't get me started! J Leukoc Biol. 2012;91:682-4.

81. Dreier R, Grässel S, Fuchs S, Schaumburger J, Bruckner P. ProMMP-9 is a specific macrophage product and is activated by osteoarthritic chondrocytes via MMP-3 or a MT1-MMP/MMP-13 cascade. Exp Cell Res. 2004;297:303-12.

82. Tinahones FJ, Coín-Aragüez L, Mayas MD, Garcia-Fuentes E, Hurtado-Del-Pozo C, Vendrell J, et al. Obesity-associated insulin resistance is correlated to adipose tissue vascular endothelial growth factors and metalloproteinase levels. BMC Physiol. 2012;12:4.

83. Zaragosi L-E, Wdziekonski B, Villageois P, Keophiphath M, Maumus $\mathrm{M}$, Tchkonia $\mathrm{T}$, et al. Activin a plays a critical role in proliferation and differentiation of human adipose progenitors. Diabetes. 2010;59:2513-21.

84. Bourlier V, Zakaroff-Girard A, Miranville A, De Barros S, Maumus $\mathrm{M}$, Sengenes $\mathrm{C}$, et al. Remodeling phenotype of human subcutaneous adipose tissue macrophages. Circulation. 2008;117:806-15.

85. Keophiphath M, Achard V, Henegar C, Rouault C, Clément K, Lacasa D. Macrophage-secreted factors promote a profibrotic phenotype in human preadipocytes. Mol Endocrinol. 2009;23:11-24.

86. Kwon E-Y, Shin S-K, Cho Y-Y, Jung UJ, Kim E, Park T, et al. Time-course microarrays reveal early activation of the immune transcriptome and adipokine dysregulation leads to fibrosis in visceral adipose depots during diet-induced obesity. BMC Genom. 2012;13:450.

87. Schram K, Sweeney G. Implications of myocardial matrix remodeling by adipokines in obesity-related heart failure. Trends Cardiovasc Med. 2008;18:199-205.

88. Tsochatzis EA, Papatheodoridis GV, Archimandritis AJ. Adipokines in nonalcoholic steatohepatitis: from pathogenesis to implications in diagnosis and therapy. Mediat Inflamm. 2009;2009:831670.

89. Shoelson SE, Herrero L, Naaz A. Obesity, inflammation, and insulin resistance. Gastroenterology. 2007;132:2169-80.

90. Virtue S, Vidal-Puig A. Adipose tissue expandability, lipotoxicity and the Metabolic Syndrome-an allostatic perspective. Biochim Biophys Acta. 2010;1801:338-49. 